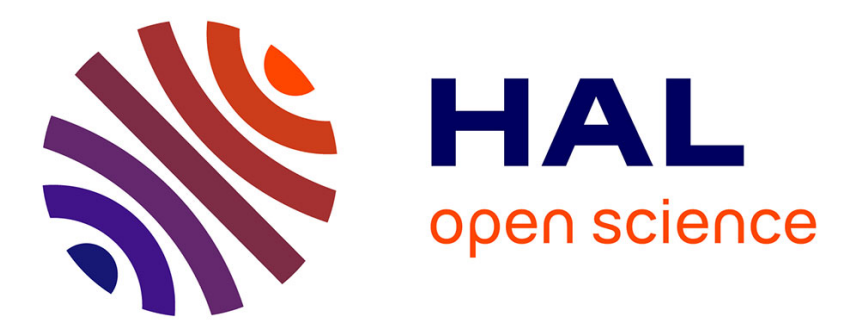

\title{
Synthetic Aperture Radar Image Classification via Mixture Approaches
}

Vladimir Krylov, Josiane Zerubia

\section{To cite this version:}

Vladimir Krylov, Josiane Zerubia. Synthetic Aperture Radar Image Classification via Mixture Approaches. IEEE International Conference on Microwaves, Communications, Antennas and Electronic Systems (COMCAS), Nov 2011, Tel Aviv, Israel. inria-00625551

\section{HAL Id: inria-00625551 \\ https://hal.inria.fr/inria-00625551}

Submitted on 21 Sep 2011

HAL is a multi-disciplinary open access archive for the deposit and dissemination of scientific research documents, whether they are published or not. The documents may come from teaching and research institutions in France or abroad, or from public or private research centers.
L'archive ouverte pluridisciplinaire HAL, est destinée au dépôt et à la diffusion de documents scientifiques de niveau recherche, publiés ou non, émanant des établissements d'enseignement et de recherche français ou étrangers, des laboratoires publics ou privés. 


\title{
Synthetic Aperture Radar Image Classification via Mixture Approaches
}

\author{
Vladimir A. Krylov and Josiane Zerubia \\ EPI Ariana, INRIA/I3S, 2004 route des Lucioles, BP93, 06902 Sophia Antipolis Cedex, France \\ email: Vladimir.Krylov@inria.fr, Josiane.Zerubia@inria.fr
}

\begin{abstract}
In this paper we focus on the fundamental synthetic aperture radars (SAR) image processing problem of supervised classification. To address it we consider a statistical finite mixture approach to probability density function estimation. We develop a generalized approach to address the problem of mixture estimation and consider the use of several different classes of distributions as the base for mixture approaches. This allows performing the maximum likelihood classification which is then refined by Markov random field approach, and optimized by graph cuts. The developed method is experimentally validated on high resolution SAR imagery acquired by Cosmo-SkyMed and TerraSAR-X satellite sensors.
\end{abstract}

Index Terms - Synthetic aperture radar, remote sensing, high resolution, classification, finite mixtures, generalized gamma distribution.

\section{INTRODUCTION}

The recent progress in sensor and antenna construction enabled the remotely sensed satellite imagery to become a widely available and attractive source of information. Satellite imagery finds its daily applications in various fields such as ecology, meteorology, oceanography, cartography, natural risk management and many others [1]. After optical data, the most common type of satellite remotely sensed imagery is registered by the synthetic aperture radar (SAR) sensors and constellations [2]. As active imaging systems operating in the domain of microwaves, SAR systems have several key advantages, such as insensitivity to sun-illumination and meteorological conditions. Whereas the first advantage may not seem very critical since it can be overcome by synchronizing correctly the orbit of an optical satellite sensor, the second advantage is indeed crucial when dealing with areas that suffered from natural disasters such as fires, hurricanes and volcano eruptions (and, therefore, are covered by smoke and/or clouds) or working with mountain and tropical areas regularly covered by clouds. On the other hand, SAR image processing poses several specific challenges: image corruption introduced by speckle and the variation in scattering coefficient with incidence angle. Speckle is an inherent phenomenon of the active imagery caused by the interference of coherent wavefronts [2]. In case of the modern satellite SAR systems, the target ground-range resolution of which can go up to submetric values, the understanding of the registered images is severely complicated by speckle. Therefore, in order to exploit the significant modern satellite SAR sensors' potential to ecological [3] and civil protection [4] applications the radar imagery needs to undergo speckle-specific image processing.

Image classification is one of the fundamental SAR processing problems [2], [5] and on its accurate and fast solution rely further application-oriented problems, such as target retrieval [1], [2] or multi-temporal change detection [6]. The problem of classification lies in assigning to each pixel of a SAR image a label which tells which landcover class has been observed at the corresponding location. Classical examples of classes include vegetation, urban, agricultural, water and road, which, given a specific classification purpose and the available data, can be merged together or split into further subclasses. The challenge in this classification problem is that the solution algorithm needs to, first, automatically estimate the number of landcover classes, and, then, distribute the pixel labels so as to verify some criterion. Much research has been conducted to derive successful solution approaches for this problem starting from the most general $K$-means and $K$ nearest neighbors algorithms [7] to SAR-specific techniques developed for polarimetric SAR imagery in [8], [9]. These techniques can report good results in some cases, however, the estimation of the number of landcover classes may sometimes be estimated incorrectly bringing to critical misclassification issues. For this reason a lot of work has been done on a simplified problem of supervised classification that poses the same pixel labeling problem under the assumption that the number of landcover classes is known a priori and for each class some certain amount of learning data is available [7]. This supervised classification problem is of significant importance since its adequate solution can automatize the work of a human operator after he or she performed the classification routine once.

Various interesting approaches have been proposed to address the supervised SAR image classification problem and, notably, the statistical algorithms that are based on explicit probability density function (PDF) models, such as, e.g., those developed in [5], [10]-[12]. These methods propose different PDFs to model the statistics of SAR amplitudes originating from homogeneous regions. Accurate classification/segmentation results can be obtained with these models in many cases, see [2], [5], [13]. However, an important limitation of these methods is given by the necessity to use statistically homogeneous classes in order to preserve the model adequacy. This limitation becomes even more critical when high resolution imagery is considered since at submetric/metric 
resolutions various materials can be visually appreciated and distinguished. This results in high level of heterogeneity within typically considered landcover classes. To address this limitation we suggest the use of PDF mixture distributions for the landcover classes which enables to explicitly take into account the impact of distinct materials and typologies on the statistics of each class.

In this paper, we focus on the problem of SAR classification using solely the single-channel amplitude data and not the complex-valued and/or full polarimetric data. This is in fact an important data typology because several image products provided by modern high resolution satellite SAR systems are geocoded ellipsoid-corrected amplitude (intensity) images, and because several earlier coarser resolution sensors (e.g., ERS) primarily used this modality.

The remainder of the paper is organized as follows. In Section II we present the overview of PDF models developed in the literature to address the problem of SAR statistics modeling and advocate the use of some of those for mixture approaches. In Section III we introduce a statistical method for the estimation of PDF mixtures from the training data. In Section IV we present the supervised classification approach that combines the PDF mixture models and the Markov random field approach for regularization. In Section V we present experiments on real high resolution SAR images obtained by the COSMO-SkyMed X-band satellite constellation and the TerraSAR-X X-band sensor. Finally, in Section VI we draw conclusions and discuss the obtained results.

\section{StATisticAl MODELS FOR SAR AMPLitUde DATA}

In this section we first recall the derivation of the most basic Rayleigh distribution for the amplitude SAR signal. This serves to give an idea how and based on what assumptions the SAR-specific statistical models can be developed. Then, we proceed to a brief overview of more specialized state-of-theart SAR-statistics models. Finally, we reason about the choice of PDF models to be employed within mixture approaches that are the central part of this paper.

\section{A. Basic statistical SAR amplitude model}

A standard model of the complex signal statistics for the case of singlelook SAR is derived as follows: The emitted signal is backscattered by a given ground area, being illuminated by a singlelook SAR sensor. We assume that the number of scatterers is large, and the scatterers are independent and small (compared to the ground area), the scattering instantaneous phases are independent of the amplitudes and uniformly distributed in $[0,2 \pi]$, and there is no single scatterer dominating the scene [2], [12]. We denote by $z$ the complex signal received by the SAR sensor from the ground area corresponding to a given pixel, so that:

$$
z=x+i y=\sqrt{v} \exp (i \theta)
$$

where $x, y, v$ and $\theta$ are the real part, the imaginary part, the intensity, and the phase of the complex signal, respectively. The model assumes the presence of a finite set of $n$ independent scattering entities in the observed area, thus interpreting $z$ as the result of the interference of the corresponding contributions. This interference phenomenon motivates the usual noiselike granular aspect of SAR images, known as speckle [2]. In particular, assuming the number of scatterers to be large, according to the central limit theorem, the real and imaginary parts of the backscattered signal are assumed to be jointly Gaussian. As a matter of fact they turn out to be independent, zero-mean Gaussian random variables with equal variances, thus yielding an exponential distribution for the signal intensity and a Rayleigh distribution for the signal amplitude (we call $r=\sqrt{v}$ the amplitude) [2]:

$$
p(r)=2 \lambda r \exp \left(-\lambda r^{2}\right)
$$

where $\lambda$ is a unknown distribution intensity parameter. Here $r, v \geqslant 0$, their PDFs are zero on $(-\infty, 0)$ and from now on we shall explicitly define their PDFs only on $[0,+\infty)$. However, real SAR amplitude data often present significantly nonRayleigh empirical distributions by, for instance, exhibiting heavier distribution tails and, thus, requiring a more accurate PDF characterization. We present some further SAR-specific PDF models in the next subsection.

\section{B. SAR amplitude model overview}

In the last decades, a number of methods have been proposed for modeling SAR amplitude PDFs, including generic nonparametric and explicit parametric models. Nonparametric methods, e.g., Parzen window estimator [16], support vector machines [17], do not assume any specific analytical model for the unknown PDF, thus providing a higher flexibility, although usually involving manual specification of internal architecture parameters [16]. Parametric methods postulate a given mathematical model for each PDF and present the PDF estimation problem as a parameter estimation problem. Empirical PDF models, including lognormal [2], Weibull [2], Fisher [5] and, recently, the generalized Gamma distribution (GND) [15], have been reported to accurately model amplitude SAR images with different heterogenous surfaces. Several theoretical models, such as Nakagami [2], generalized Gaussian Rayleigh (GGR) [11], heavy-tailed Rayleigh [12], $\mathcal{K}$ [10] $(\mathcal{K}$ root for amplitudes), have been derived from specific physical hypotheses for SAR images with different properties. Most of the above distributions are presented in Table I.

Nevertheless, most parametric families turned out to be accurate only for specific backscatter patterns, i.e., land cover typologies [2], [5], [11], making the choice of a single optimal SAR amplitude parametric PDF model a challenging task. This issue becomes especially important in case of high resolution imagery which presents characteristic statistical heterogeneity pattern. To solve this problem, we propose to consider mixture approaches as a way to combine the descriptive power of the existing models and fuse them into a single statistically flexible approach. The key question to be addressed is the choice of the PDF families which can be used to describe distributions of the mixture components (we will refer to this collection of PDFs as dictionary). In this paper we will consider several dictionaries: First, we consider the previously proposed model of Gamma PDFs that was developed in [18]. We adopt it, 
TABLE I

Dictionary $\mathcal{D}$ OF PDF FAMILIES WITH THEIR CORRESPONDING MOLC EQUATIONS. HeRE $\Gamma(\cdot)$ IS THE GAMma FUNCTION, $K_{\alpha}(\cdot)$ THE $\alpha$ Th ORDER MODIFIED BESSEL FUNCTION OF THE SECOND KIND, $J_{0}(\cdot)$ IS THE ZERO-TH ORDER BESSEL FUNCTION OF THE FIRST KIND, $\Psi(\nu, \cdot)$ THE $\nu$ TH ORDER POLYGAMMA FUNCTION [14] AND $G_{\nu}(\cdot)$ ARE THE SPECIFIC INTEGRAL FUNCTIONS FOR GGR [11]

\begin{tabular}{|c|c|c|}
\hline Family & Probability density function & MoLC equations \\
\hline $\begin{array}{c}\text { Generalized Gamma (GГD) } \\
{[15]} \\
\end{array}$ & $\begin{array}{c}f_{\nu, \kappa, \sigma}(r)=\frac{|\nu|}{\sigma \Gamma(\kappa)}\left(\frac{r}{\sigma}\right)^{\kappa \nu-1} \exp \left[-\left(\frac{r}{\sigma}\right)^{\nu}\right] \\
\nu \neq 0, \quad \kappa, \sigma>0, \quad r \geqslant 0\end{array}$ & $\begin{array}{c}k_{1}=\Psi(0, \kappa) / \nu+\ln \sigma \\
k_{j}=\Psi(j-1, \kappa) / \nu^{j}, j=2,3\end{array}$ \\
\hline Weibull [2] & $\begin{array}{c}f_{\eta, \mu}(r)=\frac{\eta}{\mu^{\eta}} r^{\eta-1} \exp \left[-\left(\frac{r}{\mu}\right)^{\eta}\right] \\
\eta, \mu>0, \quad r \geqslant 0\end{array}$ & $\begin{array}{c}k_{1}=\ln \mu+\eta^{-1} \Psi(0,1) \\
k_{2}=\eta^{-2} \Psi(1,1)\end{array}$ \\
\hline Nakagami [2] & $\begin{array}{c}f_{L, \lambda}(r)=\frac{2}{\Gamma(L)}(\lambda L)^{L} r^{2 L-1} \exp \left[-\lambda L r^{2}\right] \\
L, \lambda>0, \quad r \geqslant 0\end{array}$ & $\begin{array}{c}2 k_{1}=\Psi(0, L)-\ln \lambda-\ln L \\
4 k_{2}=\Psi(1, L)\end{array}$ \\
\hline $\begin{array}{l}\text { Fisher } \\
\text { [5], [13] }\end{array}$ & $\begin{array}{c}f_{\mu, L, M}(r)=\frac{\Gamma(L+M)}{\Gamma(L) \Gamma(M)} \frac{[C r]^{L}}{r[1+C r]^{L+M}} \\
C=\frac{L}{M \mu}, \quad \mu, L, M>0, \quad r>0\end{array}$ & $\begin{array}{c}k_{1}=\ln \mu+(\Psi(0, L)-\ln L)-(\Psi(0, M)-\ln M) \\
k_{j}=\Psi(j-1, L)+(-1)^{j} \Psi(j-1, M), j=2,3\end{array}$ \\
\hline $\begin{array}{l}K \text {-root distribution } \\
{[10]}\end{array}$ & $\begin{array}{c}f_{\mu, L, M}(r)=\frac{4}{\Gamma(L) \Gamma(M)} r^{L+M-1} C^{L+M} K_{M-L}(2 C r), \\
C=\left(\frac{L M}{\mu}\right)^{1 / 2}, \quad \mu>0,0<L<M, \quad r \geqslant 0\end{array}$ & $\begin{array}{c}2 k_{1}=\ln \mu+\Psi(0, L)+\Psi(0, M)-\ln L M \\
2^{j} k_{j}=\Psi(j-1, L)+\Psi(j-1, M), j=2,3\end{array}$ \\
\hline $\begin{array}{l}\text { Generalized Gaussian - } \\
\text { Rayleigh (GGR) [11] }\end{array}$ & $\begin{array}{c}f_{\lambda, \gamma}(r)=\frac{\gamma^{2} r}{\lambda^{2} \Gamma^{2}(\lambda)} \int_{0}^{\pi / 2} \exp \left[-(\gamma r)^{\frac{1}{\lambda}}\left(|\cos \theta|^{\frac{1}{\lambda}}+|\sin \theta|^{\frac{1}{\lambda}}\right)\right] d \theta \\
\lambda, \gamma>0, \quad r \geqslant 0\end{array}$ & $\begin{aligned} k_{1} & =\lambda \Psi(0,2 \lambda)-\ln \gamma-\lambda G_{1}(\lambda)\left[G_{0}(\lambda)\right]^{-1} \\
k_{2} & =\lambda^{2}\left[\Psi(1,2 \lambda)+\frac{G_{2}(\lambda)}{G_{0}(\lambda)}-\left(\frac{G_{1}(\lambda)}{G_{0}(\lambda)}\right)^{2}\right]\end{aligned}$ \\
\hline $\begin{array}{l}\text { Heavy-tailed Rayleigh } \\
\qquad[12]\end{array}$ & $\begin{array}{c}f_{\alpha, \gamma}(r)=r \int_{0}^{+\infty} \rho \exp \left[-\gamma \rho^{\alpha}\right] J_{0}(r \rho) d \rho \\
\alpha, \gamma>0, \quad r \geqslant 0\end{array}$ & $\begin{array}{c}\alpha k_{1}=(\alpha-1) \Psi(0,1)+\ln \gamma 2^{\alpha} \\
k_{2}=\alpha^{-2} \Psi(1,1)\end{array}$ \\
\hline
\end{tabular}

however, to the amplitude SAR imagery by replacing gamma PDFs with Nakagami, since Nakagami-Gamma is the same physical model and Gamma is its intensity version, whereas Nakagami PDF is employed in the amplitudes domain [2]. We stress that the presented in this paper Nakagami-based mixture approach is the generalization of the one developed in [18] to arbitrary number of mixture components, the estimation of which is feasible thanks to the approach presented in Section III. Second, we consider the mixtures of GГDs as a natural extension of the Nakagami-mixture model, since Nakagami represents a particular case of a GГD PDF [15]. Furthermore, this PDF seems to be especially appropriate to be a candidate for the mixture-based approaches since it also includes several other PDF families, such as Weibull, gamma, lognormal, that are known to well fit the SAR data [2] as special cases [15]. Finally, we consider the exhaustive dictionary $\mathcal{D}$ of all the distributions in Table I. Therefore, below we consider the following three distinct mixture approaches:

- Nakagami mixtures;

- GГD-mixtures;

- $\mathcal{D}$-mixtures.

We stress, that the question of choosing a particular mixture dictionary can be solved differently, thus adopting the classifier to a specific problem. The considered types of mixtures can be thought of as examples. And whatever the decision about the mixture dictionary, the mixture estimation can be performed via the general estimation approach presented in the next section.

\section{Mixture estimation}

In this section, we present the outline of dictionary-based stochastic expectation maximization (DSEM) which was ini- tially developed in [19]. We employ it here as a general approach for mixture estimation in the context of SAR image statistical modeling.

We develop a model which is based on the assumption that the distribution of grey levels of a SAR amplitude image can be described as a finite mixture distribution [20]. For each landcover class $\omega_{m}, m=1, \ldots, M$, where $M$ gives the number of target landcover classes, we assume that the training samples of $\omega_{m}$ are independent and identically distributed random variables, drawn from a mixture PDF with $K$ components:

$$
p(r)=\sum_{i=1}^{K} P_{i} p_{i}(r), \quad r \geqslant 0,
$$

where $p_{i}(\cdot)$ is the $i$-th mixture component and $\left\{P_{i}\right\}$ is a set of mixing proportions, i.e., $\sum_{i=1}^{K} P_{i}=1$ and $0 \leqslant P_{i} \leqslant 1$ for $i=1, \ldots, K$. We recall, that the underlying idea of the approach is to have each component of the mixture corresponding to one of the landcover subclasses present in each class. Therefore, each component $p_{i}(\cdot)$ is drawn from a finite dictionary $\mathcal{D}$ of SAR-specific distinct parametric PDFs $f_{j}\left(r \mid \theta_{j}\right)$, parameterized by $\theta_{j}$. We stress, that dictionary $\mathcal{D}$ may consist of arbitrary absolutely continuous PDFs concentrated on $\mathbb{R}^{+}$. Assuming that observations are quantized on the levels $\{0,1, \ldots, Z-1\}$ (it corresponds to the radiometric resolution of the acquisition [2], which is typically $8 \div 16$ bits per pixel, or, $256 \div 65536$ possible intensity values for each observation), we will denote by $h(z), z=0, \ldots, Z-1$, the amplitudes histogram restricted solely to the training samples of the considered class.

As discussed in [19], considering the variety of estimation 
approaches for finite mixtures, the appropriate choice for this particular estimation problem is the iterative stochastic expectation maximization (SEM) scheme [21]. Instead of adopting the maximum likelihood estimates as the classical SEM scheme suggests [21], since such estimates might be unfeasible for some families in the considered PDF dictionary, DSEM employs the Method of Log-Cumulants (MoLC) [5] for parameter estimation of each component. The MoLC approach has been demonstrated to be a generally feasible and effective PDF estimation tool [5], [15], [19]. By resorting to the integral Mellin transform [5], the MoLC equations relate the unknown PDF parameters with $\kappa_{1}, \kappa_{2}$ and $\kappa_{3}$ :

$$
\left\{\begin{array}{l}
\kappa_{1}=E\{\ln r\} \\
\kappa_{j}=E\left\{\left(\ln r-\kappa_{1}\right)^{j}\right\}
\end{array}, \quad j=2,3,\right.
$$

which are the $1^{\text {st }}, 2^{\text {nd }}, 3^{\text {rd }}$ order logarithmic cumulants, respectively. The MoLC equations have a single solution for any observed values of log-cumulants for all PDFs in $\mathcal{D}$ (see Table I). The solutions for the MoLC equations can be given either by analytical solutions or approximated by trivial numerical procedures, see [19].

To automatically estimate the number of mixture components we employ the procedure that consists of initializing SEM with $K^{0}=K_{\max }$, and then allowing components to be eliminated from the mixture during the DSEM iterative process, once their priors become too small, thus decreasing $K$.

Thus, each $t$-th iteration of DSEM goes as follows:

- E-step: compute, for each greylevel $z$ and $i$-th component, the posterior probability estimates corresponding to the current PDF estimates, i.e. $z=0, \ldots, Z-1$ :

$$
\tau_{i}^{t}(z)=\frac{P_{i}^{t} p_{i}^{t}(z)}{\sum_{j=1}^{K^{t}} P_{j}^{t} p_{j}^{t}(z)}, \quad i=1, \ldots, K^{t} ;
$$

- S-step: sample a component label $s^{t}(z) \in\left\{1, \ldots, K^{t}\right\}$ of each greylevel $z$ according to the current estimated posterior probability distribution $\left\{\tau_{i}^{t}(z): i=1, \ldots, K^{t}\right\}$, $z=0, \ldots, Z-1$;

- MoLC-step: for the $i$-th mixture component, compute the following histogram-based estimates of the mixture proportions and the first three log-cumulants:

$$
\begin{gathered}
P_{i}^{t+1}=\frac{\sum_{z \in Q_{i t}} h(z)}{\sum_{z=0}^{Z-1} h(z)}, \quad \kappa_{1 i}^{t}=\frac{\sum_{z \in Q_{i t}} h(z) \ln z}{\sum_{z \in Q_{i t}} h(z)}, \\
\kappa_{b i}^{t}=\frac{\sum_{z \in Q_{i t}} h(z)\left(\ln z-\kappa_{1 i}^{t}\right)^{b}}{\sum_{z \in Q_{i t}} h(z)}, \quad i=1, \ldots, K^{t},
\end{gathered}
$$

where $b=2,3$ and $Q_{i t}=\left\{z: s^{t}(z)=i\right\}$ is the set of grey levels assigned to the $i$-th component by the S-step; then, solve the corresponding MoLC equations (see Table I) for each parametric family $f_{j}\left(\cdot \mid \theta_{j}\right)$ in the dictionary, thus computing the resulting MoLC estimate $\theta_{i j}^{t}$;

- K-step: for each $i=1, \ldots, K^{t}$, if $P_{i}^{t+1}$ is below a given threshold $\alpha$, eliminate the $i$-th component and update $K^{t+1}$;

- Model Selection-step: for the $i$-th mixture component, compute the log-likelihood of each estimated PDF $f_{j}\left(\cdot \mid \theta_{i j}^{t}\right)$ according to the data assigned to the $i$-th component:

$$
L_{i j}^{t}=\sum_{z \in Q_{i t}} h(z) \ln f_{j}\left(z \mid \theta_{i j}^{t}\right), \quad i=1, \ldots, K^{t+1},
$$

and define $p_{i}^{t+1}(\cdot)$ as the estimated PDF $f_{j}\left(\cdot \mid \theta_{i j}^{t}\right)$ yielding the highest value of $L_{i j}^{t}$, where $j$ is the index to go through all PDFs in $\mathcal{D}$.

The sequence of estimates $\Theta_{t}$ generated by SEM converges to a unique stationary distribution, and the maximum likelihood estimate of the mixture parameters is asymptotically equivalent to the mathematical expectation of this stationary distribution. This behavior has been proved under suitable assumptions [21], which may or may not hold strictly depending on the contents of the considered mixture dictionary (and it is not the case for the dictionary $\mathcal{D}$ ). However, we recall that SEM, compared to the classical EM or other deterministic estimators, was specifically designed to improve the exploratory properties of EM in case of multimodal likelihood function [21].

From the practical point of view, the observed convergence rate is satisfactory: adequate estimates are typically obtained within first $T=300$ iterations. In rare cases some underestimation of $K$ may be observed, which, however, is not very critical. Threshold on the K-step of DSEM can be set arbitrarily given it is sufficiently small, and in our case we used $\alpha=0.005$.

\section{SUPERVISED CLASSIFICATION OF SAR IMAGES}

The problem of classification consists in attributing to each pixel of the considered SAR image a label assigning it to one of the $M$ thematic classes. Working in the mainframe of the supervised classification we consider some training pixels to be available for each of the $M$ classes. First, we perform the learning of the statistical properties of each class by estimating mixture PDFs on the training pixels, thus obtaining PDFs $p_{i}(r), i=1, \ldots, M$, via the algorithm developed in Section III. To do so the developed estimator is run separately for each class using the learning data. Once the PDF estimates are obtained the first most primitive classification map can be constructed by assigning to each pixel a label corresponding to one of $M$ PDFs reporting the highest value. This would give a pure maximum-likelihood (ML) classification strategy.

Yet in many cases the pure ML result is unsatisfactory and is considered too noisy for visual interpretation. There are several main sources of noise in such classification: registration errors, the maximum likelihood approach assumption and the assumption of pixel independence (involved in definition of mixture PDFs). To improve the visual quality of classification various regularization techniques might be employed: morphology, penalization or hierarchical/multiscale approaches, see [7]. Here we will employ the penalization strategy given by Markov random fields (MRFs) in the form of second-order isotropic Potts model [22] in order to improve the classification robustness to speckle. The basic idea behind this approach is to consider the classification map at each pixel and to penalize the current configuration proportional 
(with a certain weight) to the amount of neighboring pixels (only 8 neighbors are considered) that have been assigned to another class (i.e., have another class label). The employed here MRF parameter estimation is classical and we refer the reader to some earlier works for more details [22], [23]. In order to maximize the classification criterion that takes into account both the PDF estimates and the MRF-penalties an efficient iterative graph cuts-based technique is employed [24]. Notice, that the level of MRF-penalization, that is defined by a single weight parameter, can significantly affect the resulting classification. Therefore, the value of the weight parameter has to be carefully chosen so as to avoid oversmoothing. In the experiment reported below we have employed the automatic MRF-weight estimation approach developed in [23].

\section{EXPERIMENTS}

In this section we consider the applications of the developed classification mixture-based approaches to the imagery obtained by two modern SAR sensors: COSMO-SkyMed X-band satellite constellation operated by the Italian Space Agency (ASI) and TerraSAR-X X-band satellite sensor operated by the German Aerospace Center (DLR) and EADS Astrium. The considered imagery is a single-polarization high resolution GEC (geocoded ellipsoid-corrected) product in the amplitude domain. In the following experiments we analyze and compare qualitatively (by presenting the classification maps) as well as quantitatively (by reporting the obtained classification accuracies) the performance of the following classification approaches: $K$-nearest neighbors (KNN) method [7], GГDbased classification (here a single GГD PDF was employed for each class distribution, see [15]), and the developed in this paper mixture approaches based on Nakagami, GND and $\mathcal{D}$ PDFs. In the following, some of the classification maps are omitted for brevity, whereas the numerical accuracy results for all methods are presented in tables. In all the experiments, the employed learning areas of about 50x50 pixels for each target class were taken on separate images (parts of the same acquisitions not overlapping with analyzed parts).

The first set of experiments was performed on a VV polarization, $6.5 \mathrm{~m}$ ground resolution, 2.66-look TerraSAR$\mathrm{X}$ (C) EADS Astrium GmbH, 2008) image acquired over Sanchagang, China. The application was to epidemiological monitoring on a river-flooded area and the target classes were: water, wet soil and dry soil. The exhaustive ground truth map for this image was constructed based on partial expert ground truth and completed by visual interpretation. Figure 1 presents the classification results obtained with KNN and the considered mixture-based SAR statistics models: Nakagamimixtures, GГD-mixtures and $\mathcal{D}$-mixtures. The presented classification maps are referenced to the ground truth map, i.e. present in white the misclassified areas. All of the approaches were joined with the MRF model as described in Section IV. Table II reports the estimated number of components $K^{*}$ and the obtained class and overall accuracies. We observe that $\mathcal{D}$-mixtures approach slightly outperforms the GTD-mixtures model, which makes perfect sense since dictionary $\mathcal{D}$ is a collection of several PDF families, of which GГD is part.
The Nakagami-mixtures model's fit is worse due to a lower flexibility of the parametric PDF model; we recall that Nakagami (and, respectively, gamma) PDFs are special cases of GCD corresponding to $\nu=2(\nu=1)$, see Table I. The KNN method (with the parameter $K$ estimated via crossvalidation, see [7]) outperforms the Nakagami-mixtures based classification but is well behind the better adopted $\mathcal{D}$-mixtures and GCD-mixtures approaches. From the computational point of view, the classification maps were obtained in: $T_{\mathcal{D}}=$ $89 s, T_{\mathrm{G} \Gamma \mathrm{D}}=24 s, T_{\text {Nakagami }}=22 s$ on an Intel Core 2 Duo $1.83 \mathrm{GHz}, 1 \mathrm{~Gb}$ RAM, WinXP system.

The second set of experiments was performed on a $\mathrm{HH}$ polarization, $2.5 \mathrm{~m}$ ground resolution, single-look CosmoSkyMed (c)ASI, 2009) image acquired over Port-au-Prince, Haiti. This image was taken several months before the winter 2010 earthquake at Haiti and is employed as the first image of a larger sequence of acquisitions destined to track the post-earthquake urban area changes in Port-au-Prince. The classification problem is devoted to urban area detection and the thematic classes were: water, urban and land. The nonexhaustive ground truth map for this image was constructed by an expert. Figure 2 presents the classification results obtained with the KNN and the two mixture-based SAR models: $\mathcal{D}$ mixtures and GCD-mixtures. Table III reports $K^{*}$ estimates and the obtained class and overall accuracies. We observe that the $\mathcal{D}$-mixtures approach, again, slightly outperforms the GTD-mixtures model, which is, this time, bitten by the KNN method. We notice that the higher classification accuracies reported on this image are due to the nonexhaustive ground truth map used for accuracy estimation, which did not contain many class-transition areas where most of the misclassification occurs.

\section{CONCLUSIONS AND DISCUSSION}

In this paper we have introduced a unified approach for the mixture estimation for SAR amplitude statistics. We have considered several candidate PDF dictionaries for the mixture components and demonstrated the efficiency of their application to the problem of high resolution SAR image supervised classification. The experimental analysis with high resolution SAR images registered by different sensors and used in the context of different classification problems demonstrated a high potential of the mixture-based models in the problem of SAR image classification.

Several important observations can be drawn regarding the reported classification results. First, the improvement of using mixture approaches as compared to single PDF models is appreciable. To understand that it suffices to analyze the comparative performance of the single PDF GГD and mixturebased classification approaches. Second, we care to point out a comparatively small performance improvement of $\mathcal{D}$ mixtures approach compared to GCD-mixtures. Adding to that the appreciably heavier computational burden presented by $\mathcal{D}$ approach, one gets the conclusion that the dictionary has to be chosen very carefully: small enough to avoid excessive complexity of the mixture-model and yet large enough to provide the sufficient flexibility. 


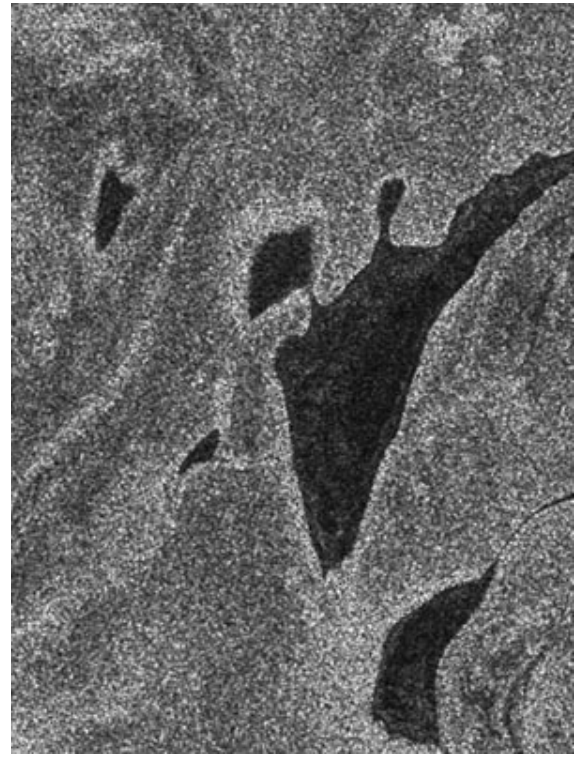

(a) River flood zone, VV polarization

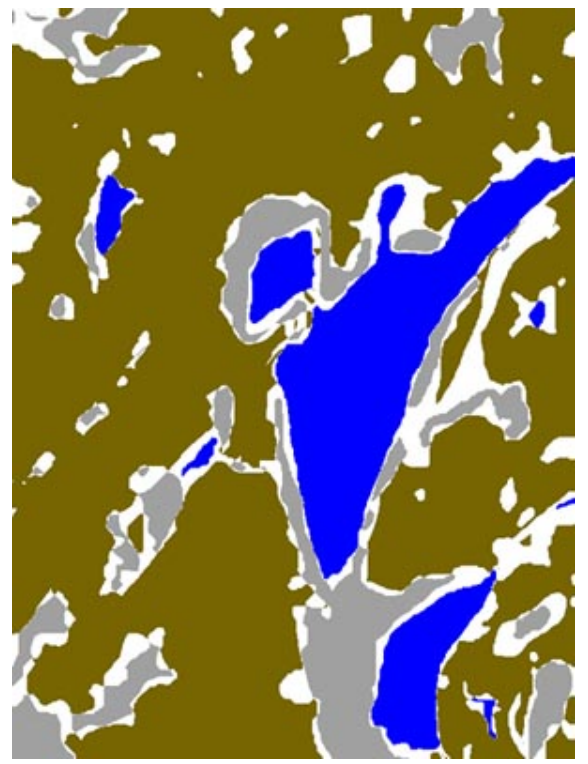

(d) $\mathcal{D}$-mixtures classification

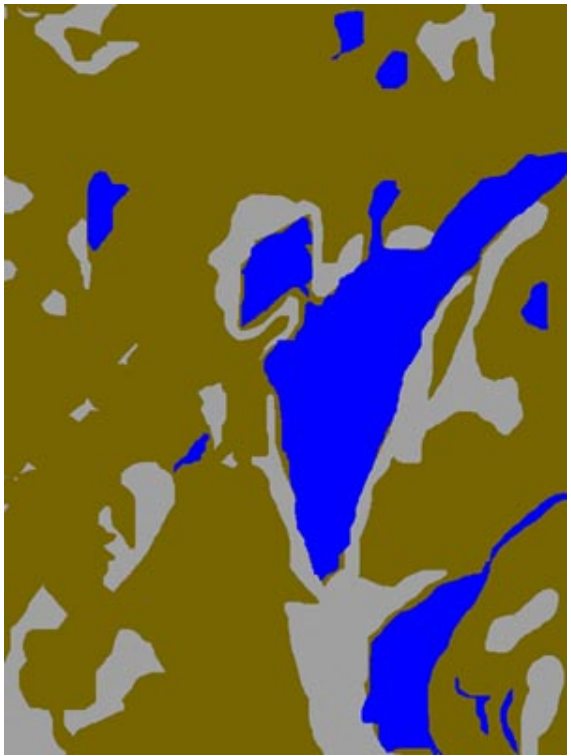

(b) Ground truth

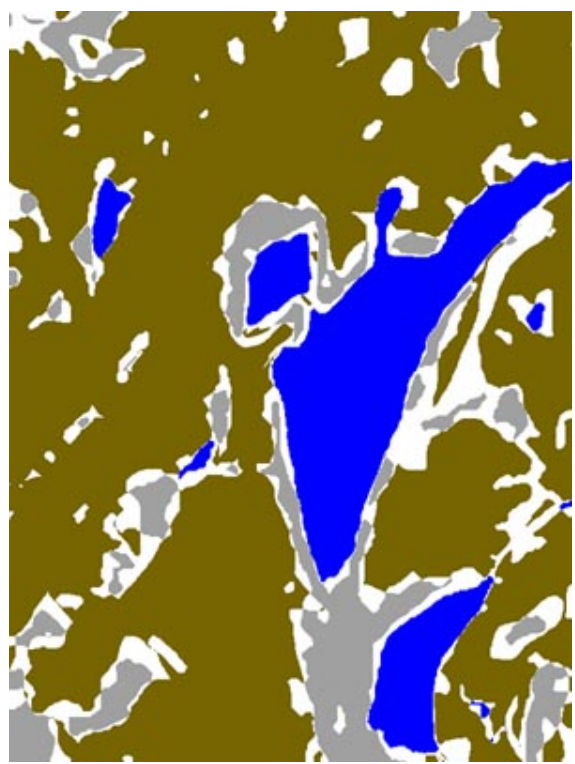

(e) GГD-mixtures classification

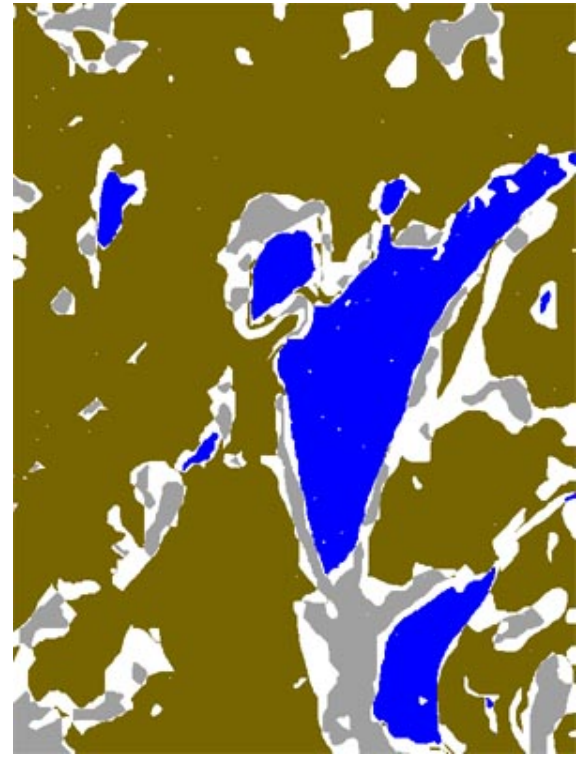

(c) KNN classification

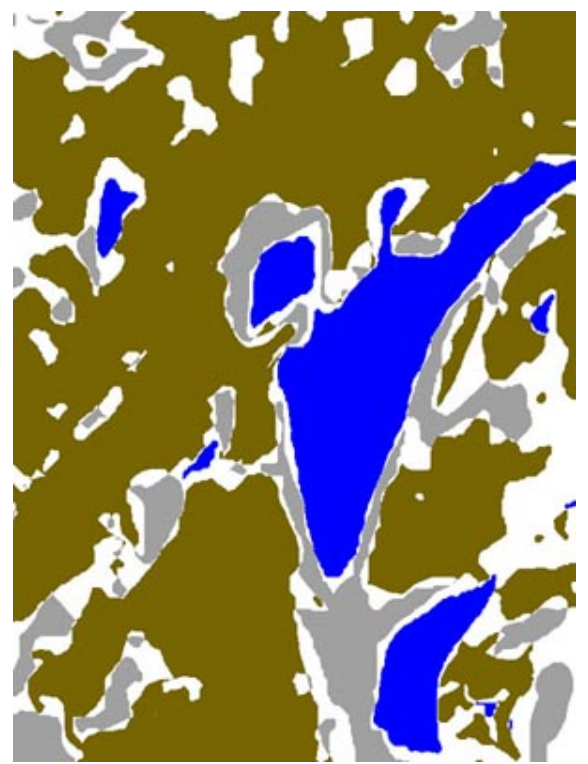

(f) Nakagami-mixtures classification

Fig. 1. Classification of the TerraSAR-X image (C)EADS Astrium GmbH) of Sanchagang, China, with three target classes: water (blue), wet soil (brown), and dry soil (grey). (a) Initial 600x800 pixels image, (b) Ground truth, and classification maps obtained using (c) KNN, (d) $\mathcal{D}$-mixtures, (e) GГD-mixtures and (f) Nakagami-mixtures approaches (here white pixels represent misclassification).

TABLE II

CLASSIFICATION RESULTS OBTAINED ON THE SANCHAGANG IMAGE: BY CLASS AND OVERALL ACCURACIES ALONG WITH NUMBER OF COMPONENTS $K$ ESTIMATES FOR THE MIXTURE-BASED METHODS.

\begin{tabular}{|c||c|c|c|c|c|c||c|}
\hline \multirow{2}{*}{ Classification method } & \multicolumn{2}{|c|}{ Water } & \multicolumn{2}{c|}{ Wet soil } & \multicolumn{2}{c|}{ Dry soil } & Overall \\
\cline { 2 - 8 } & Accuracy & $K$ & Accuracy & $K$ & Accuracy & $K$ & accuracy \\
\hline \hline KNN & $90.77 \%$ & - & $87.12 \%$ & - & $68.46 \%$ & - & $\mathbf{8 2 . 9 5 \%}$ \\
\hline GГD & $89.02 \%$ & - & $83.52 \%$ & - & $71.07 \%$ & - & $\mathbf{8 1 . 8 8 \%}$ \\
\hline GГD-mixtures & $88.55 \%$ & 2 & $90.31 \%$ & 3 & $76.49 \%$ & 3 & $\mathbf{8 7 . 0 4 \%}$ \\
\hline $\mathcal{D}$-mixtures & $88.67 \%$ & 2 & $91.06 \%$ & 2 & $81.34 \%$ & 3 & $\mathbf{8 8 . 0 9 \%}$ \\
\hline Nakagami-mixtures & $88.92 \%$ & 2 & $84.97 \%$ & 2 & $72.51 \%$ & 3 & $\mathbf{8 2 . 1 4 \%}$ \\
\hline
\end{tabular}




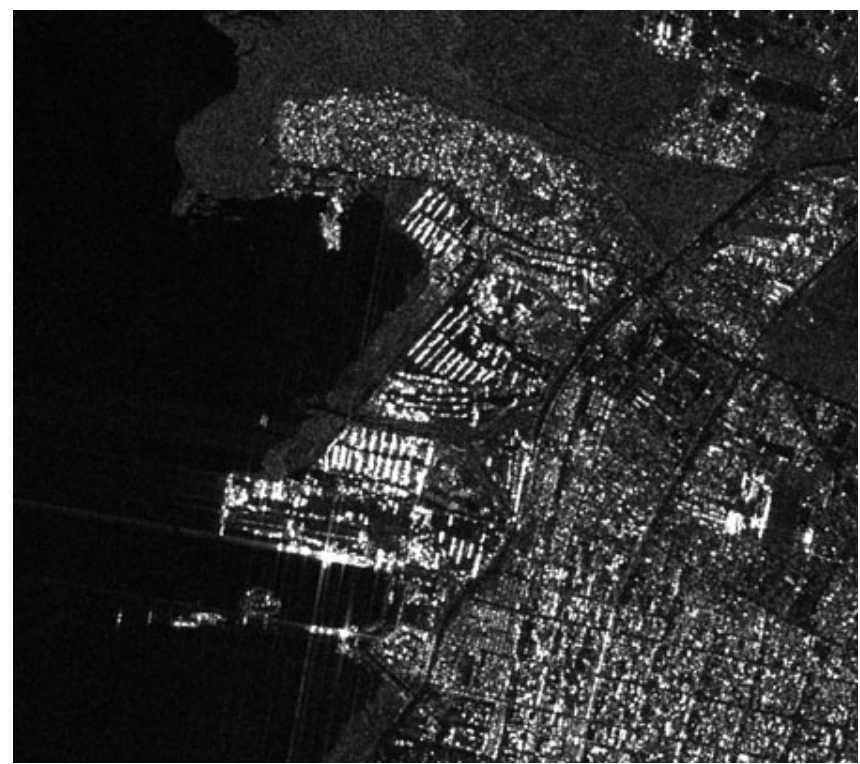

(a) Port area of Port-au-Prince, HH polarization

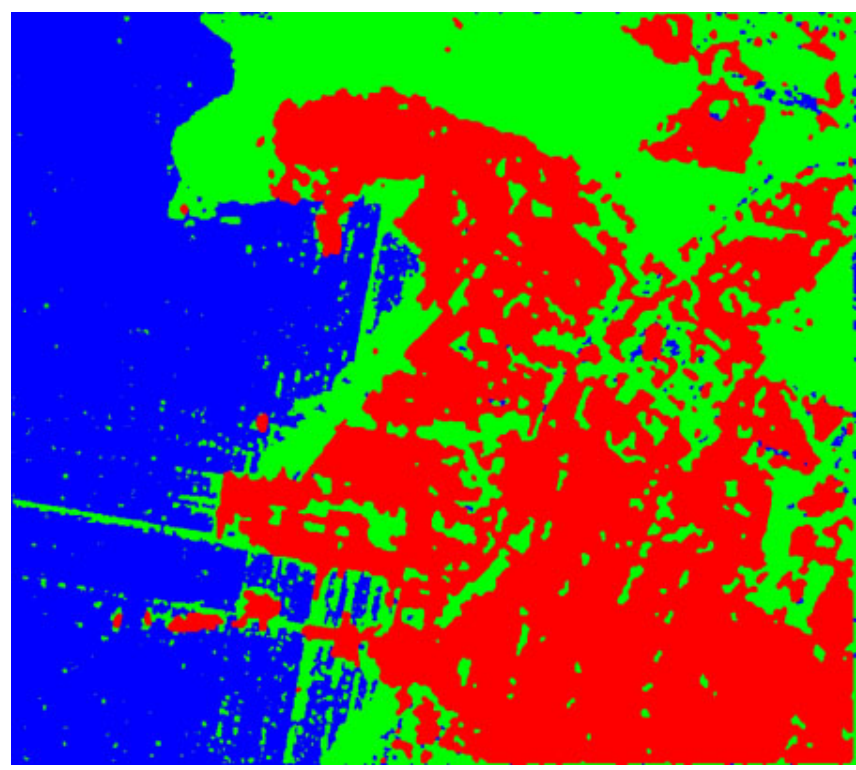

(c) GГD-mixtures classification

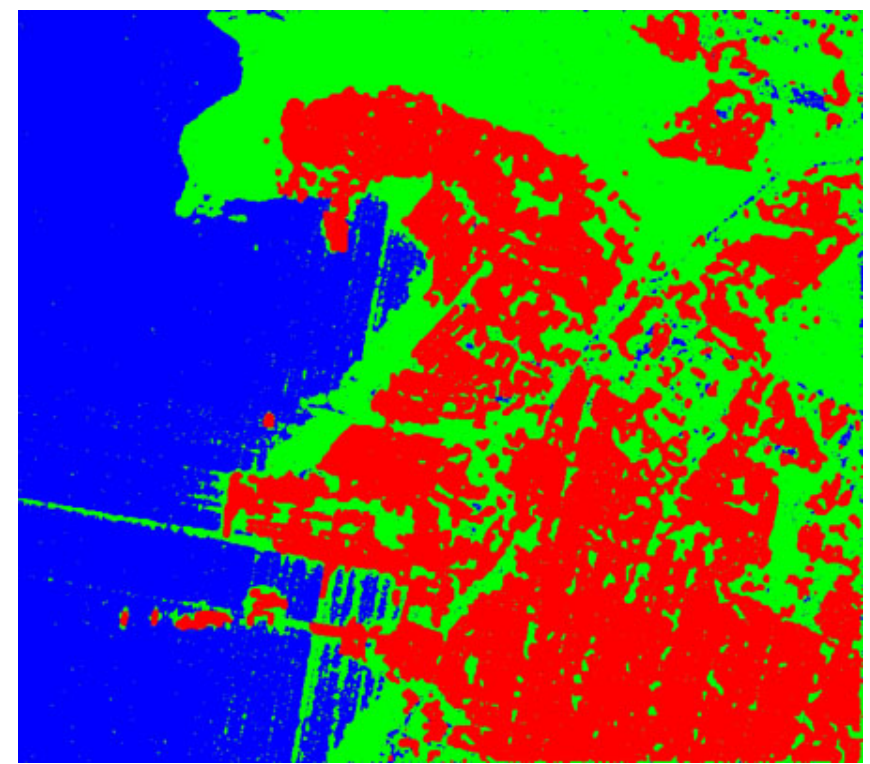

(b) KNN classification

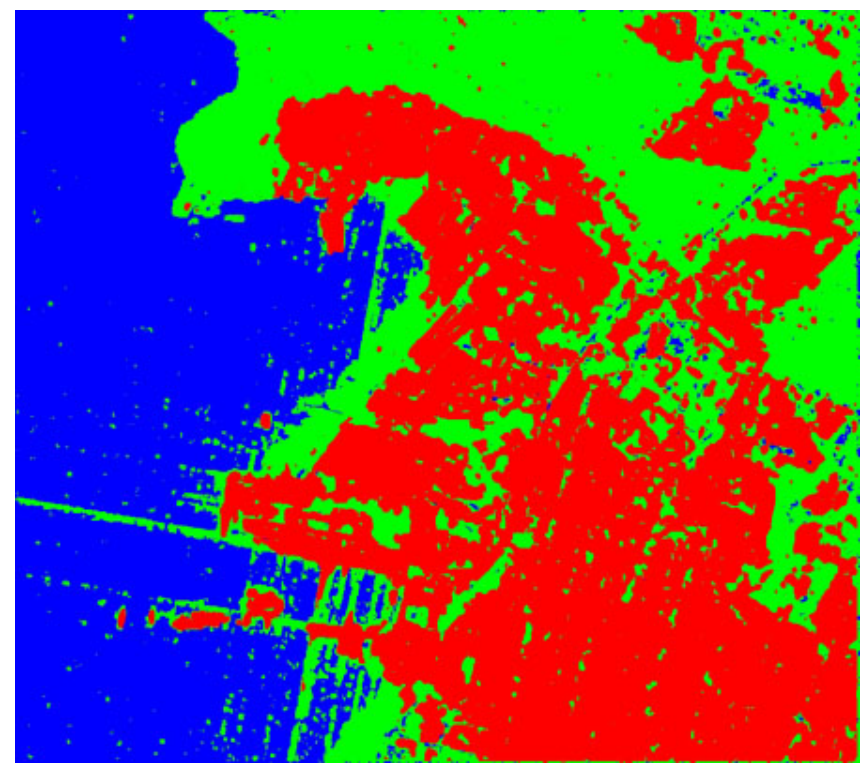

(d) $\mathcal{D}$-mixtures classification

Fig. 2. Classification of the Cosmo-SkyMed image (CASI) of Port-au-Prince, Haiti, with three target classes: water (blue), land (green), urban areas (red). (a) Initial 900x800 pixels image, and classification maps obtained using (b) KNN, (c) GГD-mixtures and (d) $\mathcal{D}$-mixtures approaches.

TABLE III

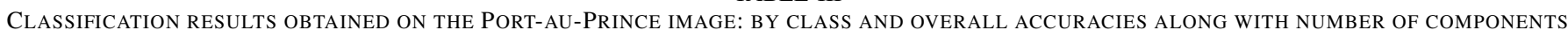
$K$ ESTIMATES FOR THE MIXTURE-BASED METHODS.

\begin{tabular}{|c||c|c|c|c|c|c||c|}
\hline \multirow{2}{*}{ Classification method } & \multicolumn{2}{|c|}{ Water } & \multicolumn{2}{c|}{ Urban } & \multicolumn{2}{c|}{ Land } & Overall \\
\cline { 2 - 8 } & Accuracy & $K$ & Accuracy & $K$ & Accuracy & $K$ & accuracy \\
\hline \hline KNN & $98.88 \%$ & - & $97.95 \%$ & - & $98.72 \%$ & - & $\mathbf{9 8 . 5 1 \%}$ \\
\hline GГD & $99.12 \%$ & - & $95.16 \%$ & - & $98.17 \%$ & - & $\mathbf{9 7 . 1 7 \%}$ \\
\hline GГD-mixtures & $98.35 \%$ & 2 & $98.81 \%$ & 3 & $98.49 \%$ & 2 & $\mathbf{9 8 . 4 4 \%}$ \\
\hline $\mathcal{D}$-mixtures & $98.33 \%$ & 2 & $99.06 \%$ & 3 & $98.86 \%$ & 2 & $\mathbf{9 8 . 7 2 \%}$ \\
\hline Nakagami-mixtures & $98.42 \%$ & 2 & $96.77 \%$ & 2 & $97.90 \%$ & 3 & $\mathbf{9 7 . 8 5 \%}$ \\
\hline
\end{tabular}


In the introduction we have mentioned that we consider solely the single-channel amplitude SAR data. This does not mean that the developed approach cannot be employed when dealing with data in different modalities. First, the intensity extension is straightforward by replacing the amplitude models by the intensity PDFs in the dictionary. Second, the developed approach can be directly extended to multichannel/polarimetric SAR data in the way developed in [23].

\section{ACKNOWLEDGMENT}

The authors would like to thank Aurélie Voisin (INRIA Sophia Antipolis, France) for her helpful assistance with experiments, and Gabriele Moser and Sebastiano B. Serpico (Univ. of Genoa, Italy) for their helpful comments and assistance in preparing this paper. We would also like to thank the Italian Space Agency for providing the COSMOSkyMed (CSK $囚)$ image of Port-au-Prince (COSMO-SkyMed Product - (C)ASI - Agenzia Spaziale Italiana - 2009. All Rights Reserved). The employed TerraSAR-X image of Sanchagang was taken from http://www.infoterra.de/, where it is available for free testing (EADS Astrium $\mathrm{GmbH}, 2008$ ).

\section{REFERENCES}

[1] J. Richards and X. Jia., Remote sensing digital image analysis, SpringerVerlag, Berlin, 4th edition, 2006.

[2] C. Oliver and S. Quegan, Understanding Synthetic Aperture Radar Images, SciTech, Raleigh, NC, USA, 2nd edition, 2004.

[3] E. S. Kasischke, J. M. Melack, and M. C. Dobson, "The use of imaging radars for ecological applications. a review," Remote Sens. Environ., vol. 59, no. 2, pp. 141-156, 1997.

[4] G. Boni, F. Castelli, L. Ferraris, N. Pierdicca, S. B. Serpico, and F. Siccardi, "High resolution COSMO/SkyMed SAR data analysis for civil protection from flooding events," in Proceedings of IGARSS, Barcelona, Spain, 2007, pp. 6-9.

[5] C. Tison, J.-M. Nicolas, F. Tupin, and H. Maitre, "A new statistical model for Markovian classification of urban areas in high-resolution SAR images," IEEE Trans. Geosci. Remote Sens., vol. 42, no. 10, pp. 2046-2057, 2004.

[6] G. Moser and S. B. Serpico, "Unsupervised change detection from multichannel SAR data by Markovian data fusion," IEEE Trans. Geosci. Remote Sens., vol. 47, no. 7, pp. 2114-2128, 2009.

[7] C. M. Bishop, Pattern Recognition and Machine Learning, Springer, New York, 2006.
[8] P. R. Kersten, J.-S. Lee, and T. L. Ainsworth, "Unsupervised classification of polarimetric synthetic aperture radar images using fuzzy clustering and EM clustering," IEEE Trans. Geosci. Remote Sens., vol. 43 , no. 3, pp. 519-527, 2005.

[9] K. Ersahin, I.G. Cumming, and R.K Ward, "Segmentation and classification of polarimetric SAR data using spectral graph partitioning," IEEE Trans. Geosci. Remote Sens., vol. 48, no. 1, pp. 164-174, 2010.

[10] E. Jakeman and P. N. Pusey, "A model for non-Rayleigh sea echo," IEEE Trans. Antennas Propagat., vol. 24, pp. 806-814, 1976.

[11] G. Moser, J. Zerubia, and S. B. Serpico, "SAR amplitude probability density function estimation based on a generalized Gaussian model," IEEE Trans. Image Process., vol. 15, no. 6, pp. 1429-1442, 2006.

[12] E. E. Kuruoglu and J. Zerubia, "Modelling SAR images with a generalization of the Rayleigh distribution," IEEE Trans. Image Process., vol. 13 , no. 4 , pp. 527-533, 2004.

[13] F. Galland, J.-M. Nicolas, H. Sportouche, M. Roche, F. Tupin, and P. Refregier, "Unsupervised synthetic aperture radar image segmentation using Fisher distributions," IEEE Trans. Geosci. Remote Sens., vol. 47, no. 8, pp. 2966-2972, 2009.

[14] M. Abramowitz and I. Stegun, Eds., Handbook of Mathematical Functions, Dover, New York, 1964.

[15] H.-C. Li, W. Hong, Y.-R. Wu, and P.-Z. Fan, "On the empirical-statistical modeling of SAR images with generalized gamma distribution," IEEE J. Sel. Top. Signal Process., vol. 5, no. 3, pp. 386-397, Jun. 2011.

[16] R. O. Duda, P. E. Hart, and D. G. Stork, Pattern classification, Wiley Interscience, New York, 2001.

[17] P. Mantero, G. Moser, and S. B. Serpico, "Partially supervised classification of remote sensing images using SVM-based probability density estimation," IEEE Trans. Geosci. Remote Sens., vol. 43, no. 3, pp. 559$570,2005$.

[18] J.-M. Nicolas and F. Tupin, "Gamma mixture modeled with "second kind statistics": application to SAR image processing," in Proceedings of Internat. Geosci. Remote Sens. Symposium, Toronto, Canada, 2002, pp. 2489-2491.

[19] V. A. Krylov, G. Moser, S. B. Serpico, and J. Zerubia, "Enhanced dictionary-based SAR amplitude distribution estimation and its validation with very high-resolution data," IEEE Geosci. Remote Sens. Lett., vol. 8, no. 1, pp. 148-152, Jan. 2011.

[20] M. A. F. Figueiredo and A. K. Jain, "Unsupervised learning of finite mixture models," IEEE Trans. Patt. Anal. Mach. Intell., vol. 24, no. 3 , pp. 381-396, 2002.

[21] G. Celeux, D. Chauveau, and J. Diebolt, "Stochastic versions of the EM algorithm: an experimental study in the mixture case," J. Statist. Comp. Sim., vol. 55, no. 4, pp. 287-314, 1996.

[22] J. Besag, "On the statistical analysis of dirty pictures," J. Royal Stat. Soc. B, vol. 48, pp. 259-302, 1986.

[23] V. A. Krylov, G. Moser, S. B. Serpico, and J. Zerubia, "Supervised highresolution dual-polarization SAR image classification by finite mixtures and copulas," IEEE J. Sel. Top. Signal Process., vol. 5, no. 3, pp. 554 566, Jun. 2011.

[24] Y. Boykov, O. Veksler, and R. Zabih, "Efficient approximate energy minimization via graph cuts," IEEE Trans. Patt. Anal. Mach. Intell., vol. 20, no. 12, pp. 1222-1239, Nov. 2001. 